\title{
Asymptomatic Carotid Stenosis and Risk of Stroke (ACSRS) study: what have we learned from it?
}

\author{
Kosmas I. Paraskevas ${ }^{1}$, Andrew N. Nicolaides ${ }^{2}$, Stavros K. Kakkos ${ }^{3}$ \\ ${ }^{1}$ Department of Surgery, Central Clinic of Athens, Athens, Greece; ${ }^{2}$ Department of Surgery, University of Nicosia Medical School, Nicosia, Cyprus; \\ ${ }^{3}$ Department of Vascular Surgery, University of Patras Medical School, Patras, Greece \\ Contributions: (I) Conception and design: KI Paraskevas; (II) Administrative support: AN Nicolaides, SK Kakkos; (III) Provision of study materials or \\ patients: All authors; (IV) Collection and assembly of data: KI Paraskevas; (V) Data analysis and interpretation: All authors; (VI) Manuscript writing: \\ All authors; (VII) Final approval of manuscript: All authors. \\ Correspondence to: Andrew N. Nicolaides, MS, FRCS, PhD (Hon). Professor of Vascular Surgery, Department of Surgery, University of Nicosia \\ Medical School, Nicosia, Cyprus. Email: anicolaides1@gmail.com.
}

\begin{abstract}
The Asymptomatic Carotid Stenosis and Risk of Stroke (ACSRS) study is the largest natural history study on patients with 50-99\% asymptomatic carotid stenosis (ACS). It included 1,121 ACS individuals with a follow-up between 6 and 96 months (mean: 48 months). During the last 15 years, several important ACSRS substudies have been published that have contributed significantly to the optimal management of ACS patients. These studies have demonstrated that specific baseline clinical characteristics and ultrasonic plaque features after image normalization (namely carotid plaque type, gray scale median, carotid plaque area, juxtaluminal black area without a visible echogenic cup, discrete white areas in an echolucent part of a plaque, silent embolic infarcts on brain computed tomography scans, a history of contralateral transient ischemic attacks/strokes) can independently predict future ipsilateral cerebrovascular events. The ACSRS study provided proof that by use of a computer program to normalize plaque images and extract plaque texture features, a combination of features can stratify patients into various categories depending on their stroke risk. The present review will discuss the various reported predictors of future ipsilateral cerebrovascular events and how these characteristics can be used to calculate individual stroke risk.
\end{abstract}

Keywords: Asymptomatic carotid stenosis (ACS); stroke risk; carotid endarterectomy (CEA); Asymptomatic Carotid Stenosis and Risk of Stroke (ACSRS)

Submitted Jan 07, 2020. Accepted for publication Feb 13, 2020.

doi: $10.21037 /$ atm.2020.02.156

View this article at: http://dx.doi.org/10.21037/atm.2020.02.156

\section{Introduction}

The Asymptomatic Carotid Stenosis and Risk of Stroke (ACSRS) study was a multicenter study conducted under the auspices of the International Union of Angiology (1-3). Until this day, ACSRS is the biggest natural history study on patients with 50-99\% asymptomatic carotid stenosis (ACS) including a total of 1,121 patients with a follow-up between 6 and 96 months (mean: 48 months). The conception of the ACSRS study took place in 1996, following the report of the Asymptomatic Carotid Atherosclerosis Study (ACAS) (4). Patient recruitment began in 1998 and ended in $2002(1-3)$.

\section{Study design}

All centers participating in ACSRS had a non-invasive vascular laboratory equipped with a color duplex facility. Each ACSRS center examined >500 patients/year, and employed staff that was experienced in the investigation and management of patients with extracranial cerebrovascular disease consisting of a neurologist, a vascular physician/ vascular surgeon and a radiologist (1-3). By screening new attendees to their practice, each participating center was 
able to identify and recruit on average 15 individuals with ACS (1-3).

Patients were eligible for recruitment to ACSRS if: (I) they had 50-99\% ACS in relation to the carotid bulb diameter [European Carotid Surgery Trial (ECST) method], (II) they reported no previous ipsilateral cerebral or retinal ischemic (CORI) events and (III) they demonstrated no neurological abnormalities on examination (1-3). Patients with previous contralateral CORI events were also considered for inclusion provided they had been asymptomatic for $>6$ months at the time of recruitment. In patients demonstrating bilateral ACS, the side which exhibited more severe stenosis was recorded as ipsilateral (i.e., the study artery) (1-3).

A detailed patient history and physical examination were recorded at baseline to make sure that patients were truly asymptomatic upon study entry. Upon admission to the study, a duplex ultrasound scanning was performed to grade internal carotid artery (ICA) stenosis. This duplex examination was repeated every 6 months thereafter to monitor progression of ICA stenosis (1-3). All patients were followed up with the aim to identify specific subgroups at high (or low) risk for future CORI events. Each patient was reviewed at all visits by both a neurologist and a radiologist to record clinical information as well as the rate of ICA stenosis progression (1-3).

\section{Duplex examination}

Velocities were recorded at the site of maximum ICA stenosis at the center of the common carotid artery lumen, with the beam of the ultrasound at a $60^{\circ}$ angle to the arterial wall and to the direction of flow (1-3). A disadvantage of absolute velocity measurements is that they can underestimate (e.g., in the presence of cardiac arrhythmia) or overestimate ACS (e.g., in the presence of severe contralateral disease). Consequently, the radiologists performing the Duplex examinations in each centre were trained to use a combination of absolute velocity measurements and velocity ratios (1-3). Using these velocities and velocity ratios, they could express the percentage diameter stenosis in relation to both the distal normal ICA [North American Symptomatic Carotid Endarterectomy Trial (NASCET) method] and to the bulb diameter (ECST method). Measurement of arterial flow in the vertebral arteries flow (cephalad, reversed or absent) was also noted. The complete duplex examination was recorded on S-VHS videotape and was submitted to the coordinating center in order for image analysis and quality control to be performed centrally.

Plaque images were obtained in B-Mode (black and white), and color or power Doppler. The latter methods were used to outline juxtaluminal black areas (JBA) of plaque. The depth was minimized in order for the plaques to occupy a large part of the image. The ultrasound beam was at $90^{\circ}$ to the arterial wall. The overall gain was adjusted to minimize but not completely abolish noise. By minimizing (but not completely abolishing) noise, the radiologists managed not to reduce the gain too much to lose low-intensity features in the plaque. This also ensured that there remained a black area without noise (which represented blood in the vessel lumen) and could be used for image normalization. Finally, the position of the probe was adjusted so that adventitia adjacent to the plaque was clearly visible as a hyperechoic band that could also be used for image normalization. This technique of image capture with ultrasound at right angles to the arterial wall was essential and has not been adequately appreciated. The reason for having the beam of ultrasound at right angles to the vessel wall is that one cannot do image analysis on plaques images obtained with the ultrasound beam at $60^{\circ}$ because the adventitia is not shown at its brightest and thus image normalization cannot be performed. Image normalization consisted of adjusting the Gray Scale Median (GSM) value of 2 reference points (blood to be 0 and adventitia to be 190) with all other values automatically being adjusted on a linear scale. This ensured that reproducible GSM measurements could be obtained if a patient was scanned on different equipment in different ambient lights and that comparable readings of GSM measurements in a serial follow-up would be available in a multicenter study.

A detailed description of image texture analysis is beyond the scope of this review and it is presented in detail elsewhere (3).

\section{Results and key messages}

The key outcomes reported in the various ACSRS substudies and preliminary reports over the course of 15 years are presented in Table 1 (5-13). A total of 1,121 patients with $50-99 \%$ ACS aged $39-89$ years (mean age: 70.0 7.7 years; $61 \%$ males) were recruited in ACSRS. Overall, 130 first ipsilateral CORI events were recorded during follow-up [59 strokes (of which 12 were fatal), 49 transient ischemic attacks (TIAs) and 22 cases of amaurosis fugax] (9). 


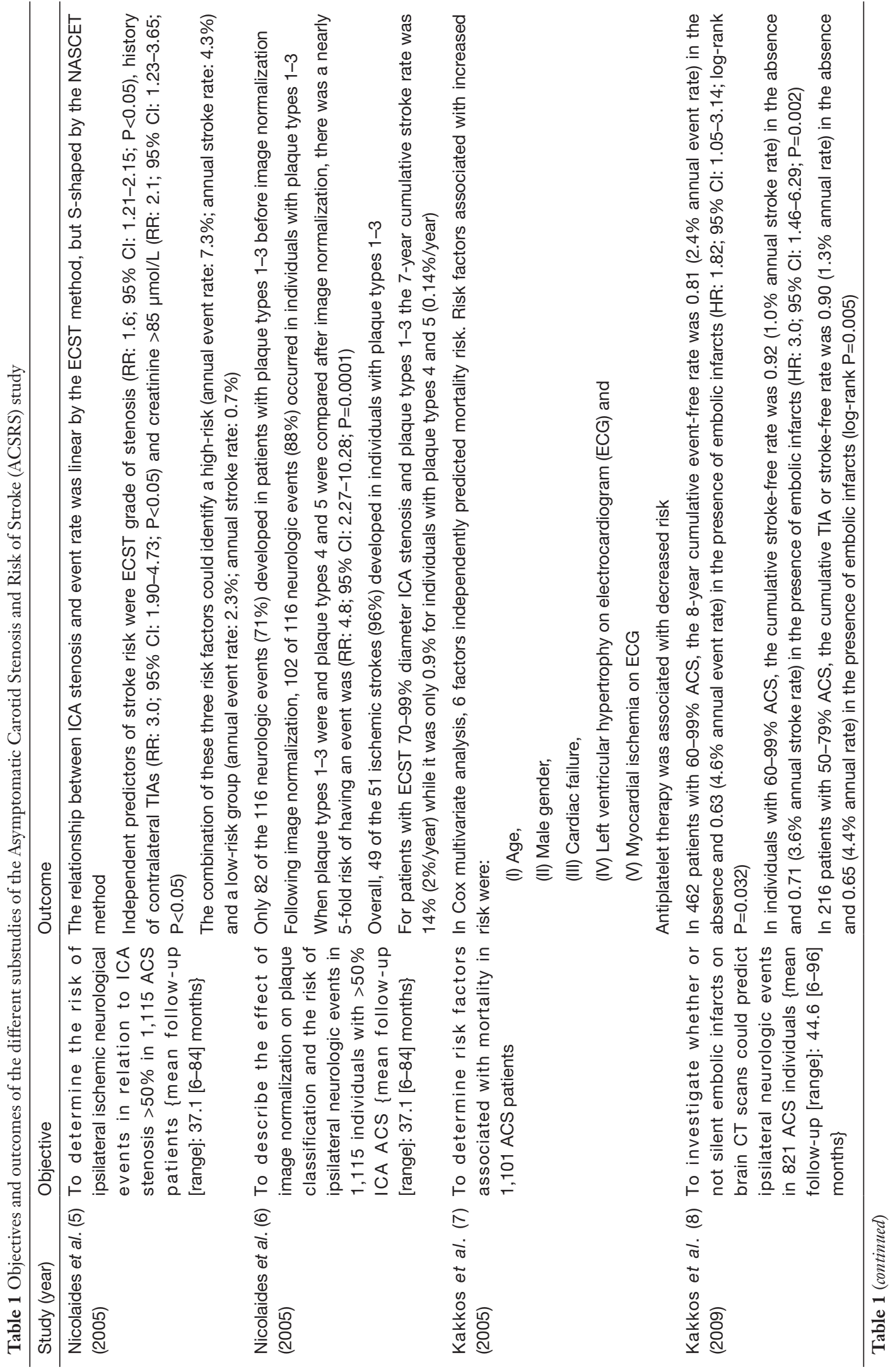




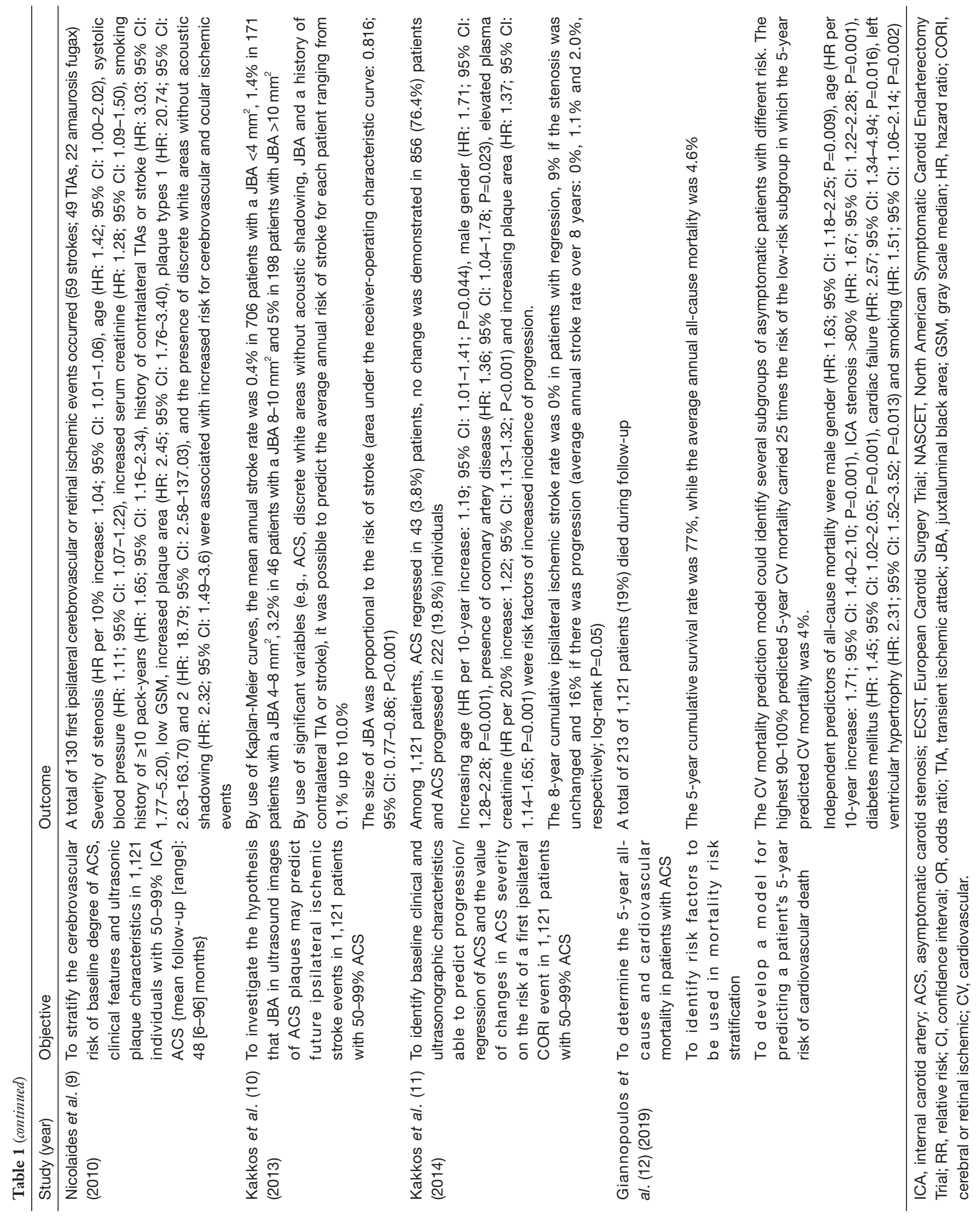




\section{Stenosis}

Ipsilateral \% ACS was recorded as mild (50-69\%) in 198 study participants, as moderate (70-89\%) in 598 individuals and as severe (90-99\%) in 325 patients (9). The cumulative 5 -year ipsilateral CORI event rate for these three ACS groups was $9 \%, 15 \%$ and $20 \%$, respectively (log-rank test $\mathrm{P}=0.009)$. This resulted in an average annual event rate of $1.8 \%, 3.0 \%$ and $4.0 \%$, respectively. Furthermore, the cumulative stroke rate over 5 years for the 3 ACS groups was $4 \%, 7 \%$ and $12 \%$, respectively (log-rank test $\mathrm{P}=0.011$ ). This resulted in an average annual stroke rate of $0.8 \%, 1.4 \%$ and $2.4 \%$, respectively (9). For $>70 \%$ ACS, the cumulative 5 -year stroke rate was $8 \%$, giving an average annual stroke rate of $1.6 \%$ (9).

Based on these results, it was concluded that the severity of stenosis was a relatively poor indicator of stroke risk with a receiver operator characteristic (ROC) area under the curve (AUC) of 0.603 [95\% confidence interval (CI): 0.5250.682] (5). In the subgroup of 325 patients with stenosis greater than $90 \%$ (ECST) the average annual stroke rate was $2.4 \%$. However, this subgroup contained only 25 of the 59 strokes (or $42 \%$ ) that occurred in the whole group. In other words, the severity of stenosis alone could not identify a high-risk group that contained the majority of strokes.

An important finding was that the ECST percentage stenosis had a linear relationship-while the NASCET percentage stenosis had an S-shaped relationship-to stroke risk (5) and as such, it could not be used in a multivariable linear logistic regression. A possible explanation for this finding is that ECST percentage stenosis correlates to the plaque volume in the bulb, while NASCET percentage stenosis correlates to lumen diameter reduction in relation to the lumen diameter of the normal distal ICA.

\section{Plaque type and stroke risk}

An important issue that has not received enough attention is that in order to successfully analyze texture features of carotid plaques, one should first do image normalization (6). This issue has been missed by international guidelines and recommendations. The majority of previous natural history studies have employed several techniques to classify plaque without first performing image normalization. Image normalization changes the appearance of plaques markedly resulting in a reclassification of a considerable percentage of them. In ACSRS, 652 (60\%) of the plaques changed category after image normalization (6). When comparing plaque types $1-3 v s$. types 4 and 5 before image normalization, the relative risk (RR) of suffering a CORI event was 1.12 (95\% CI: 0.76-1.66; $\mathrm{P}=0.45)$. In contrast, when plaque types 1-3 were compared with plaque types 4 and 5 after image normalization, the RR of a CORI event was 4.8 (95\% CI: $2.27-10.28 ; \mathrm{P}=0.0001)(6)$.

An important association is the incidence of ipsilateral ischemic stroke $v s$. both plaque type and ACS severity (6). For patients with $50-69 \%$ ACS, the stroke rate was low regardless of plaque type. For patients with $70-89 \%$ ACS, the incidence of stroke was $5.7 \%$ in individuals with plaque types $1-3$ and $0.8 \%$ in those with types 4 and 5 . Finally, for individuals with $90-99 \%$ ACS, the incidence of stroke was $7.7 \%$ in study participants with plaque types $1-3 v s .0 \%$ in those with types 4 and 5 (6). Therefore, for the 905 study participants with 70-99\% ACS, the incidence of stroke was $6.5 \%$ (47 of 724) for those with plaque types 1, 2 and 3 and only $0.55 \%$ ( 1 of 181 ) for those with plaque types 4 and 5 (RR: 11.7; 95\% CI: 1.63-84.5; $\mathrm{P}=0.003$ ) (6). Individuals with plaque types $1-3$ had a cumulative stroke rate of $14 \%$ at 7 years (or 2\%/year), while those with plaque types 4 and 5 had a cumulative stroke rate of $1 \%$ at 7 years (i.e., $0.14 \%$ per year) (6).

In ACSRS, the type of plaque determined by the software after image normalization demonstrated a clear-cut separation of stroke risk by plaque type. This finding lends further support to the importance of image normalization before image analysis and proves that plaque types 4 and 5 are associated with a low stroke risk, regardless of the degree of stenosis. Importantly, $76 \%$ of the strokes (45 of 59 ) occurred in the $38 \%$ of patients $(426$ of 1,121$)$ with type 1 and 2 plaques at baseline, while severe and fatal ipsilateral strokes occurred exclusively in patients with plaque types 1 and 2 (6). The conclusion reached was that ACS study participants with plaque types 4 and 5 following image normalization, are at low stroke risk even when the degree of stenosis is severe and therefore may not need to offered a carotid endarterectomy (CEA) (6). By contrast, patients with high degree of ACS and plaque types 1 through 3 are at increased risk and may therefore require a prophylactic CEA (6).

\section{GSM}

Prior to the ACSRS study, prospective studies from two different centers reported that hypoechoic plaques (13) or plaques with low GSM after image normalization (14) were 
associated with the development of ipsilateral ischemic strokes. The ACSRS study showed that a low GSM after image normalization strongly predicts future ipsilateral CORI events (9). The GSM was low $[<15]$ in 243 patients, moderate [15-30] in 269 and high [ $>30]$ in 609 patients. The cumulative incidence of 5 -year stroke for these 3 groups was $2 \%, 4 \%$ and $15 \%$, respectively (log-rank test $\mathrm{P}<0.001$ ), equating to an average stroke rate of $0.6 \% / y e a r$, $1.6 \% /$ year and $3.6 \% / y e a r$, respectively (9). However, the subgroup of 243 patients with GSM $<15$ contained only 31 $(53 \%)$ of the 59 strokes that occurred in the whole cohort during follow-up.

\section{Carotid plaque area}

Prospective studies from two different centers reported that carotid plaque area $(15,16)$ were associated with the development of ipsilateral ischemic strokes. The results from ACSRS showed that carotid plaque area can be used for cerebrovascular risk stratification in individuals with plaques producing $>50 \%$ stenosis (9). Plaque area was large $\left(>80 \mathrm{~mm}^{2}\right)$ in 114 individuals, intermediate $\left(40-80 \mathrm{~mm}^{2}\right)$ in 489 and small $\left(<40 \mathrm{~mm}^{2}\right)$ in 518 study participants. The cumulative incidence of 5 -year stroke for these three groups was $5 \%, 7 \%$ and $23 \%$, respectively (log-rank test $\mathrm{P}<0.001$ ), equating to an average annual stroke rate of $1.0 \%, 1.4 \%$ and $4.6 \%$, respectively (9). However, the subgroup of 114 patients with plaque area $>80 \mathrm{~mm}^{2}$ contained only $16(27 \%)$ of the 59 strokes that were recorded in the entire group during follow-up.

\section{$\mathcal{F B A}$ without a visible echogenic cup}

The finding of a JBA without a visible echogenic cap has been reported to be associated with symptomatic plaques in several cross-sectional studies (17-19). However, the various cut-off points for size of this area had not been reported. In ACSRS, JBA was classified into four groups; JBA was $<4 \mathrm{~mm}^{2}$ in 704 patients, $4-8 \mathrm{~mm}^{2}$ in 171 patients, $8-10 \mathrm{~mm}^{2}$ in 46 patients and $>10 \mathrm{~mm}^{2}$ in 198 patients (10). The cumulative incidence of 5 -year stroke in these 4 groups was $2 \%, 7 \%, 16 \%$ and $23 \%$, respectively, equating to an average stroke rate of $0.4 \%$ /year, $1.4 \%$ /year, $3.2 \%$ /year and $5.0 \% /$ year, respectively (log-rank test $\mathrm{P}<0.001)(10)$. A JBA of $\geq 8 \mathrm{~mm}^{2}$ found in 245 patients was associated with a $4.1 \%$ average annual stroke rate. This subgroup presented with $42(71 \%)$ of the 59 strokes that were recorded in the whole group during follow-up. A JBA of $\geq 8 \mathrm{~mm}^{2}$ together with a GSM $<15$ were associated with a high prevalence of symptomatic plaques and demonstrated the highest combined specificity and sensitivity for the development of hemispheric symptoms. Importantly, the cut-off value of 8 $\mathrm{mm}^{2}$ separated two groups, one with a high and one with a low prevalence of symptomatic plaques regardless of the grade of stenosis (10). These results strongly suggest that JBA can be used to identify a high-risk group which will contain most of the strokes. It also provides proof that a JBA $\geq 8 \mathrm{~mm}^{2}$ is a critical cut-off point.

\section{Discrete white areas (DWA)}

An earlier study already demonstrated that plaque heterogeneity is associated with symptomatic plaques (20) and this is often produced by the presence of DWA in hypoechoic plaques or plaque areas. These DWA do not have an associated acoustic shadow which excludes calcification and they consist of neovascularization as shown by perfusion studies using microbubble agents (21). In the ACSRS study, DWA were absent in 403 patients and in this subgroup they were associated with an average annual stroke rate of $1.2 \%$. They were present in 718 patients and associated with an average annual stroke rate of $1.8 \%$. The latter group contained 45 (76\%) of the 59 strokes that developed in the whole group during follow-up.

\section{Silent embolic infarcts on brain CT scans}

Another ACSRS sub-study investigated whether the presence of silent brain infarction on CT scans was associated with an increased stroke risk (8). In individuals with less than $60 \%$ ACS, the presence of embolic infarcts $(n=60)$ was not associated with an increased stroke risk compared with those patients $(n=299)$ who did not demonstrate silent infarcts (annual stroke rate: $2.38 \% \mathrm{vs}$. $1.66 \%$, respectively; HR: $1.14 ; 95 \%$ CI: $0.50-2.58 ; \mathrm{P}=0.65$ ). In contrast, the annual stroke rate was significantly higher in 462 patients with 60-99\% ACS in the presence of embolic infarcts compared with their absence (annual stroke rate: $3.6 \%$ vs. $1.0 \%$, respectively; HR: 3.0; 95\% CI: 1.46-6.29; $\mathrm{P}=0.002)(8)$. In multivariate logistic regression analysis with ipsilateral embolic infarcts, degree of stenosis $(<60 \%$, $60-79 \%, 80-99 \%)$, age, gender, history of myocardial infarction, arrhythmia, as well as use of antiarrhythmic medications, antiplatelet use and anticoagulant therapy 
as independent variables, silent embolic infarcts (RR: 2.0; 95\% CI: 1.1-3.8; $\mathrm{P}=0.033$ ) and degree of stenosis (RR: 1.6; $95 \% \mathrm{CI}: 1.1-2.5 ; \mathrm{P}=0.019)$ were independent predictors of ipsilateral neurologic events (8). These results suggest that silent embolic infarcts on brain CT scan could identify a high-risk group for ipsilateral CORI events (8). However, this group only contained $30 \%$ of the strokes that occurred in the entire group during follow-up.

\section{Clinical/biochemical features associated with increased risk}

A history of contralateral TIAs or stroke was the only clinical factor demonstrating a strong association with future CORI events and stroke (9). The cumulative incidence of 5 -year stroke was $6 \%$ for those individuals $(\mathrm{n}=948)$ without a history of contralateral TIA or stroke, while it was $17 \%$ for the group of 173 patients with prior symptoms (logrank test $\mathrm{P}<0.001$ ), equating to an average annual stroke rate of $1.2 \% v s .3 .4 \%$, respectively (9). Although a previous contralateral TIA or stroke is noted only in a minority of patients, it should be viewed as a feature associated with high future stroke risk, especially when combined with the high-risk plaque features discussed above.

\section{Plaque progression and regression}

In an ACSRS sub-study, several independent baseline predictors of plaque regression were identified including younger age, high grades of ACS, absence of DWA in the plaque and administration of statins (11). In contrast, high serum creatinine, male gender, lack of statin use, low grade of ACS and increased plaque area were independent baseline predictors of plaque progression (11). Of the 59 strokes that occurred, 40 (68\%) were recorded in individuals whose stenosis remained unchanged, whereas 19 (32\%) occurred in those patients with progression and 0 in those with regression (11). For the whole group, the 8 -year cumulative ipsilateral cerebral ischemic stroke rate was $0 \%$ in patients with regression, $9 \%$ if the stenosis was unchanged and $16 \%$ if there was progression (average annual stroke rates of $0 \%$, $1.1 \%$ and $2.0 \%$, respectively; log-rank $\mathrm{P}=0.05)(11)$.

\section{Calculation of individual stroke risk}

Stenosis severity, history of contralateral TIAs or stroke, a low GSM, high plaque area, JBA $\geq 8 \mathrm{~mm}^{2}$ and plaque heterogeneity resulting from DWA without acoustic shadow in hypoechoic areas were associated with the development of future ipsilateral CORI events in both univariate and multivariate analysis (9). However, in the presence of JBA as a covariate, plaque area and GSM were no longer significant independent predictors. Thus, in this latest model only degree of ACS, history of contralateral TIA or stroke, presence of DWA and JBA remained significant independent predictors (10). As a result, the risk of any patient could be calculated or obtained from specially constructed tables derived from this model. The predicted annual average stroke rate was $<1.0 \%$ (very low risk) in 734 patients, $1.0-1.9 \%$ (low risk) in $94,2.0-3.9 \%$ (moderate risk) in 134 (11\%), 4.0-5.9\% (high risk) in $125(11 \%)$ and $>6.0 \%$ (very high risk) in $34(3 \%)$ patients (10). Calculation of this risk for individual patients was provided by the software.

Patients in the ACSRS study were not on what is currently considered to be optimal medical therapy. Assuming patients are now on optimal medical therapy which is expected to reduce stroke risk by $50 \%$, then only the patients allocated to the high risk and very high risk by the ACSRS methodology, i.e., $13 \%$ of those with $>50 \%$ ECST stenosis or $17 \%$ of those with ECST stenosis $>70 \%$ (50\% NASCET) would be considered for CEA.

\section{Mortality}

A key issue when considering an intervention in an individual with ACS is life-expectancy. It is not worth considering a prophylactic CEA in an asymptomatic patient, if this individual will not survive long enough to benefit from the procedure. To this end, an ACSRS interim report was produced aiming to identify risk factors able to predict long-term mortality (7), while a more recent analysis attempted to develop a model for predicting the 5 -year risk of cardiovascular death in patients with ACS (12). Age, gender, stenosis, diabetes, cardiac failure, left ventricular hypertrophy and lack of antiplatelet therapy were independent predictors of cardiovascular mortality (12). Based on the risk prediction model that was developed, the 5 -year all-cause mortality was $<10 \%$ in $236,10-25 \%$ in 579 , $25-40 \%$ in 204 and $>40 \%$ in 102 patients. In patients with predicted 5 -year mortality $>40 \%$, the death/stroke ratio was high even in those predicted to have a 5 -year risk of stroke $>10 \%$. In fact, the majority of the patients in this subgroup died before a stroke would occur even in the presence of an unstable carotid plaque. Such patients should not be considered as candidates for CEA. 
The risk prediction model developed is unique not only because it was derived from the ACSRS, a natural history study which included $>1,100$ asymptomatic patients, but also due to the fact that-in contrast to previous studies-it provided a tool for stratifying both cardiovascular mortality and stroke risk. An interesting finding is that the predicted mortality risk is not related to the stroke risk. The predicted mortality risk remained high even in the presence of a low stroke risk group. This means that patients with low stroke risk who will not be considered for carotid surgery because of a low-grade stenosis or a stable (e.g., echogenic or calcified) plaque should not be denied aggressive risk factor modification. They are at a very high risk of a myocardial infarction, and therefore, a cardiac assessment is indicated. The authors point out that such an assessment is a unique opportunity that may never occur again in the patient's lifetime.

\section{Recent histological studies and ultrasonic texture features after image normalization}

Early histological studies have shown that in symptomatic plaques the necrotic core is twice as close to the lumen compared with asymptomatic plaques (22). A number of subsequent cross-sectional studies using ultrasound demonstrated a relationship between JBA with the presence of patient neurological symptoms $(17,19,23)$.

Correlations between ultrasonic texture features after image normalization and plaque histology have been made more recently. A low GSM is associated with a histologically unstable plaque (24), less calcification (25), low collagen content, a large lipid core, a thin fibrous cap $(24,26)$, increased inflammation and neovascularization (26). DWAs in a hypoechoic area of a plaque were associated with intraplaque haemorrhage and inflammation in one study (25) and with neovascularization, increased number of macrophages and intraplaque haemorrhage in another (26). A JBA near the luminal portion of the plaque (without a visible cap) is associated with a necrotic core located close to the lumen on histology (23), macroscopic plaque ulceration (25), decreased numbers of smooth muscle cells, large lipid core, thin fibrous cap and plaque rupture (26).

The above histological studies provide an explanation for the association of many ultrasonic plaque texture features with plaque stability or instability and they explain the ability of such features to predict future strokes as demonstrated in the ACSRS study.

\section{Discussion}

ACSRS was the largest prospective study on asymptomatic carotid patients managed with medical intervention alone. It showed that baseline clinical characteristics and ultrasonic plaque features are independent predictors of future ipsilateral CORI events.

Four important points from ACSRS have not been so far appreciated:

(I) The fact that image normalization is essential before analyzing texture features of carotid plaques;

(II) Equipment settings and plaque image capture with ultrasound at right angles to the arterial wall are similarly essential;

(III) The texture features used in the ACSRS were not data-derived. The texture features used in the ACSRS study had been found to be associated with symptomatic plaques in earlier cross-sectional studies, which validated externally their value, and

(IV) The best stroke risk stratification depends on a combination of texture features (such as JBA and DWA) together with percentage of ACS (ECST method), as well as with history of contralateral stroke/TIA.

ACSRS was unique because it did not concentrate only on one feature, but rather it demonstrated that plaque characteristics can add significantly/improve stroke risk stratification. More importantly, it provided a method for the calculation of stroke risk for each individual patient. The implications of ACSRS are that clinical and ultrasonic plaque features can be used to stratify stroke risk. This can subsequently lead to refinement of the indications for CEA. The availability of user-friendly software for image analysis ("Plaque Texture Analysis Software" by LifeQ Medical Ltd.; lifeqmedical.com) and for automatic calculation of risk may establish this method as part of routine practice in the vascular laboratory. This software can calculate stroke risk for each individual patient and may provide a report of the measurement of key texture features including the predicted annual stroke risk.

\section{Conclusions}

The ACSRS results suggest that clinical and ultrasonic plaque features can be used to stratify stroke risk and may therefore refine the indications for CEA in asymptomatic individuals. User-friendly software for image analysis is currently available and automatic calculation of stroke risk for each ACS individual may help targeting prophylactic 
CEA procedures to those asymptomatic patients most likely to develop symptoms in the future. Information derived from the ACSRS study should help clinical decisionmaking.

Some carotid plaques may continue to grow despite implementation of rigorous optimal medical therapy. There are many risk factors and/or genetic factors we are not aware of. Furthermore, there are other emerging risk factors (such as homocysteine), which we do not routinely treat. For example, individuals with polymorphism of methylenetetrahydrofolate reductase (MTHFR) and inadequate folic acid intake may develop high homocysteine levels (27). In this context, there have been 2 randomized controlled trials in the past treating homocysteine $v s$. placebo in patients with advanced coronary artery disease which were negative in terms of the coronary artery disease, but there was a significant reduction in stroke $(28,29)$. ACSRS has provided a valuable source of exploring and testing new methods of image analysis and new algorithms for calculation of stroke and mortality risk. Future studies should further explore additional pathways to provide optimal management of patients with ACS.

\section{Acknowledgments}

Funding: None.

\section{Footnote}

Provenance and Peer Review: This article was commissioned by the editorial office, Annals of Translational Medicine, for the series "Carotid Artery Stenosis and Stroke: Prevention and Treatment Part I". The article has undergone external peer review.

Conflicts of Interest: All authors have completed the ICMJE uniform disclosure form (available at http://dx.doi. org/10.21037/atm.2020.02.156). ANN has shares in LifeQ Medical Ltd. The other authors have no conflicts of interest to declare.

Ethical Statement: The authors are accountable for all aspects of the work in ensuring that questions related to the accuracy or integrity of any part of the work are appropriately investigated and resolved.

Open Access Statement: This is an Open Access article distributed in accordance with the Creative Commons
Attribution-NonCommercial-NoDerivs 4.0 International License (CC BY-NC-ND 4.0), which permits the noncommercial replication and distribution of the article with the strict proviso that no changes or edits are made and the original work is properly cited (including links to both the formal publication through the relevant DOI and the license). See: https://creativecommons.org/licenses/by-nc-nd/4.0/.

\section{References}

1. Nicolaides A, Sabetai M, Kakkos SK, et al; ACSRS Study Group. The Asymptomatic Carotid Stenosis and Risk of Stroke (ACSRS) study. Aims and results of quality control. Int Angiol 2003;22:263-72.

2. Nicolaides AN. Asymptomatic carotid stenosis and risk of stroke. Identification of a high risk group (ACSRS). A natural history study. Int Angiol 1995;14:21-3.

3. Kyriacou EC, Petroudi S, Pattichis CS, et al. Prediction of high-risk asymptomatic carotid plaques based on ultrasonic image features. IEEE Trans Inf Technol Biomed 2012;16:966-73.

4. Endarterectomy for asymptomatic carotid artery stenosis. Executive Committee for the Asymptomatic Carotid Atherosclerosis Study. JAMA 1995;273:1421-8.

5. Nicolaides AN, Kakkos S, Griffin M, et al; Asymptomatic Carotid Stenosis and Risk of Stroke (ACSRS) Study Group. Severity of asymptomatic carotid stenosis and risk of ipsilateral hemispheric ischaemic events: results from the ACSRS study. Eur J Vasc Endovasc Surg 2005;30:275-84.

6. Nicolaides AN, Kakkos SK, Griffin M, et al. Effect of image normalization on carotid plaque classification and the risk of ipsilateral hemispheric ischemic events: results from the asymptomatic carotid stenosis and risk of stroke study. Vascular 2005;13:211-21.

7. Kakkos SK, Nicolaides A, Griffin M, et al. Factors associated with mortality in patients with asymptomatic carotid stenosis: results from the ACSRS Study. Int Angiol 2005;24:221-30.

8. Kakkos SK, Sabetai M, Tegos T, et al. Asymptomatic Carotid Stenosis and Risk of Stroke (ACSRS) Study Group. Silent embolic infarcts on computed tomography brain scans and risk of ipsilateral hemispheric events in patients with asymptomatic internal carotid artery stenosis. J Vasc Surg 2009;49:902-9.

9. Nicolaides AN, Kakkos SK, Kyriacou E, et al. Asymptomatic internal carotid artery stenosis and cerebrovascular risk stratification. J Vasc Surg 
2010;52:1486-1496.e1.

10. Kakkos SK, Griffin MB, Nicolaides AN, et al; Asymptomatic Carotid Stenosis and Risk of Stroke (ACSRS) Study Group. The size of juxtaluminal hyperchoic area in ultrasound images of asymptomatic carotid plaques predicts the occurrence of stroke. J Vasc Surg 2013;57:609-618.e1.

11. Kakkos SK, Nicolaides AN, Charalambous I, et al. Predictors and clinical significance of progression or regression of asymptomatic carotid stenosis. J Vasc Surg 2014;59:956-967.e1.

12. Giannopoulos A, Kakkos S, Griffin MV, et al. Mortality risk stratification in patients with asymptomatic carotid stenosis. Vasc Invest Ther 2019;2:25-32.

13. Polak JF, Shemanski L, O'Leary DH, et al. Hypoechoic plaque at US of the carotid artery: an independent risk factor for incident stroke in adults aged 65 years or older. Cardiovascular Health Study. Radiology 1998;208:649-54.

14. Elatrozy T, Nicolaides A, Tegos T, et al. The objective characterization of ultrasonic carotid plaque features. Eur J Vasc Endovasc Surg 1998;16:223-30.

15. Spence JD, Eliasziw M, DiCicco M, et al. Carotid plaque area: a tool for targeting and evaluating vascular preventive therapy. Stroke 2002;33:2916-22.

16. Mathiesen EB, Johnsen SH, Wilsgaard T, et al. Carotid plaque area and intima-media thickness in prediction of first-ever ischemic stroke: a 10-year follow-up of 6584 men and women: the Tromso Study. Stroke 2011;42:972-8.

17. Griffin MB, Kyriacou E, Pattichis C, et al. Juxtaluminal hypoechoic area in ultrasonic images of carotid plaques and hemispheric symptoms. J Vasc Surg 2010;52:69-76.

18. Pedro LM, Pedro MM, Goncalves I, et al. Computerassisted carotid plaque analysis: characteristics of plaques associated with cerebrovascular symptoms and cerebral infarction. Eur J Vasc Endovasc Surg 2000;19:118-23.

19. Pedro LM, Fernandes e Fernandes J, Pedro MM, et al. Ultrasonographic risk score of carotid plaques. Eur J Vasc Endovasc Surg 2002;24:492-8.

20. Carra G, Visona A, Bonanome A, et al. Carotid plaque

Cite this article as: Paraskevas KI, Nicolaides AN, Kakkos SK. Asymptomatic Carotid Stenosis and Risk of Stroke (ACSRS) study: what have we learned from it? Ann Transl Med 2020;8(19):1271. doi: 10.21037/atm.2020.02.156 morphology and cerebrovascular events. Int Angiol 2003;22:284-9.

21. Shah F, Balan P, Weinberg M, et al. Contrastenhanced ultrasound imaging of atherosclerotic carotid plaque neovascularization: a new surrogate marker of atherosclerosis? Vasc Med 2007;12:291-7.

22. Bassiouny HS, Sakaguchi Y, Mikucki SA, et al. Juxtalumenal location of plaque necrosis and neoformation in symptomatic carotid stenosis. J Vasc Surg 1997;26:585-94.

23. Sztajzel R, Momjian-Mayor I, Comelli M, et al. Correlation of cerebrovascular symptoms and microembolic signals with the stratified gray-scale median analysis and color mapping of the carotid plaque. Stroke 2006;37:824-9.

24. Salem MK, Bown MJ, Choke E, et al. Identification of patients with a histologically unstable carotid plaque using ultrasonic plaque image analysis. Eur J Vasc Endovasc Surg 2014;48:118-25.

25. Mitchell CC, Stein JH, Cook TD, et al. Histopathologic Validation of Grayscale Carotid Plaque Characteristics Related to Plaque Vulnerability. Ultrasound Med Biol 2017;43:129-37.

26. Spanos K, Tzorbatzoglou I, Lazari P, et al. Carotid plaque echomorphology and its association with histopathologic characteristics. J Vasc Surg 2018;68:1772-80.

27. Panayiotou A, Nicolaides A, Griffin M, et al. Serum total homocysteine, folate, 5,10-methylenetetrahydrofolate reductase (MTHFR) 677C-->T genotype and subclinical atherosclerosis. Expert Opin Ther Targets 2009;13:1-11.

28. Lonn E, Yusuf S, Arnold MJ, et al. Heart Outcomes Prevention Evaluation (HOPE) 2 Investigators. Homocysteine lowering with folic acid and $\mathrm{B}$ vitamins in vascular disease. N Engl J Med 2006;354:1567-77.

29. Saposnik G, Ray JG, Sheridan P, et al. Heart Outcomes Prevention Evaluation 2 Investigators. Homocysteinelowering therapy and stroke risk, severity and disability: additional findings from the HOPE 2 trial. Stroke 2009;40:1365-72. 